\title{
Qualidade interna do ovo de avestruz após estocagem em temperatura ambiente e refrigerada
}

\author{
Internal quality of ostrich eggs after storage in ambient temperature and in cooling condition storage
}

\author{
Andréa Kammers Pleti ${ }^{*}$ Jair José de Lima ${ }^{\text {II }}$ Lys Mary Bileski Candido ${ }^{\mathrm{I}, \mathrm{II}}$
}

\section{RESUMO}

O objetivo deste trabalho foi avaliar as alterações da qualidade interna de três tipos de ovos de avestruzes: frescos, armazenados sob temperatura ambiente $\left(25^{\circ} \mathrm{C}\right)$ e sob temperatura de refrigeração $\left(5^{\circ} \mathrm{C}\right)$, durante três, sete, $14,21 \mathrm{e}$ 28 dias de armazenamento. Houve queda significativa na Unidade Haugh, aumento do $\mathrm{pH}$ e diminuição da viscosidade em relação ao tempo de armazenamento. Os ovos mantidos em temperatura ambiente apresentaram maiores alterações do que os ovos armazenados sob refrigeração. Houve uma crescente perda de peso durante os dias de armazenamento, tanto sob temperatura ambiente, quanto em temperatura refrigerada, porém a perda de peso foi mais evidente na temperatura ambiente.

Palavras-chave: ovo, avestruz, unidade Haugh, armazenamento, pH do albúmen.

\section{ABSTRACT}

The objective of this research was to evaluate the inside changes quality of fresh ostrich eggs and eggs stored in ambient temperature $\left(25^{\circ} \mathrm{C}\right)$ and in cooling condition storage $\left(5^{\circ} \mathrm{C}\right)$, during 3, 7, 14, 21 and 28 days after storage. There was a considerable slump at Unity Haugh, $\mathrm{pH}$ increasing and viscosity decreasing related to time storage. Eggs stored in ambienttemperature showed more alterations than the ones stored under refrigeration. There was an increasing loss of weight during the days of storage in ambient temperature and in cooling condition storage, although the loss of weight was more notable in the ambient temperature.

Key words: eggs, ostrich, unit Haugh, storage, albumen.

\section{INTRODUÇÃO}

Pela designação “ovo” entende-se o ovo de galinha em casca, sendo os demais acompanhados da indicação da espécie de que procedem (BRASIL, 1990). O ovo é um alimento de grande valor nutritivo e recomendado como alimento para uma dieta variada e equilibrada. Devido às suas características funcionais tecnológicas, é amplamente utilizado pelas indústrias nos mais diversos produtos e preparações.

O ovo de avestruz corresponde, em média, a 25 ovos de galinha. Em geral, ele mede de 12 a $18 \mathrm{~cm}$ de altura e de 10 a $15 \mathrm{~cm}$ de largura, seu peso varia de $1,0 \mathrm{~kg}$ a $2,0 \mathrm{~kg}$, e os ovos inférteis servem para o consumo humano e para indústria de alimentos (CARRER et al., 2004). No entanto, não existe ainda. Legislação sobre o ovo de avestruz. Portanto, são necessárias pesquisas para garantir o consumo com segurança.

O ovo é um alimento perecível e começa a perder sua qualidade interna imediatamente após a postura. Para retardar a velocidade do processo de perda da qualidade do ovo, devem ser utilizadas baixas temperaturas de armazenamento após a coleta (SOUZA et al., 1994). As medidas que melhor representam a qualidade interna dos ovos são a unidade Haugh e o Índice Gema (SOUZA et al., 1998). A unidade Haugh relaciona o peso do ovo (g) com a altura da clara (mm) (PEEBLES et al., 2000). O uso da unidade Haugh tem sido geralmente aceito como uma medida da qualidade do albúmen nos estudos de qualidade do ovo (EISEN et al., 1962). Embora sujeita a críticas, é considerada uma medida padrão de qualidade e tem sido usada pela

IPrograma de Pós-graduação Tecnologia de Alimentos, Universidade Federal do Paraná (UFPR), Curitiba, PR, Brasil. *Endereço para correspondência: Av. Prefeito Lothário Meissner, 632, Jd. Botânico, 80210-170, Curitiba, PR, Brasil. E-mail: andreapleti@brturbo.com.br. ${ }^{I}$ Departamento de Nutrição, UFPR, Curitiba, PR, Brasil. 
indústria avícola (WILLIANS, 1992). Um valor alto para unidades Haugh está associado a ovos de boa qualidade, e a taxa de diminuição, nas unidades Haugh, aumenta em temperaturas elevadas de armazenamento (BERARDINELLI et al., 2003).

Durante o armazenamento, o $\mathrm{pH}$ do ovo se eleva devido à perda de dióxido de carbono. $\mathrm{O}$ pH da clara, originalmente cerca de 7,9, eleva-se para 9,3 nos primeiros dias de armazenamento, mudando pouco daí em diante. À medida que o ovo é armazenado, o dióxido de carbono se difunde por meio da casca até que se equilibre com a relativamente pequena quantidade existente no ar (GRISWOLD, 1972; LINDEN \& LORIENT, 1996).

O objetivo deste trabalho foi avaliar a alteração da qualidade interna do ovo de avestruz por meio de medidas do $\mathrm{pH}$, da viscosidade, da unidade Haugh e da perda de peso durante o armazenamento em temperatura ambiente e sob refrigeração.

\section{MATERIAL E MÉTODOS}

Foram utilizados 30 ovos higienizados de avestruzes . Os ovos foram pesados em balança de precisão e distribuídos em dois grupos: grupo 1 composto por ovos armazenados em temperatura ambiente, que foi monitorada $\left(24\right.$ a $\left.28^{\circ} \mathrm{C}\right)$, e umidade relativa do ar entre 56 e $78 \%$. O grupo 2 foi composto por ovos refrigerados sob temperatura de 2 a $5^{\circ} \mathrm{C}$. As determinações foram realizadas em triplicata, e os períodos de armazenamento foram de três, sete, 14, 21 e 28 dias. A perda de peso foi calculada, subtraindo-se o peso final, após o armazenamento nos dias três, sete, 14, 21 e 28 do peso inicial, no dia da postura, e dividindo-se essa diferença pelo peso inicial. O resultado foi expresso em porcentagem. A clara e gema foram separadas, e o $\mathrm{pH}$ foi medido por meio de um pHmetro digital PG 2000 Gehaka. A viscosidade foi avaliada por meio do viscosímetro Brookfield (Modelo LVF). Devido às características da amostra, empregouse um adaptador UL (ultra low).

Para a unidade Haugh, os ovos foram quebrados sobre uma mesa especial de acrílico, onde a altura do albúmen foi medida por meio de um micrômetro. De posse dos dados de peso (g) e altura (mm), calculou-se a unidade Haugh mediante a seguinte equação:

$$
\mathrm{UH}=100 \log \left[\mathrm{H}-\frac{\sqrt{\mathrm{G}(30 \mathrm{w} 0,37-100)}}{100}+1,9\right]
$$

$\mathrm{HU}=$ Unidade Haugh, $\mathrm{H}=$ Altura do albúmen em milímetros, $\mathrm{W}=$ Peso do ovo em gramas, $\mathrm{G}=$ constante gravitacional de valor 32 (BRANT et al., 1951).

O delineamento experimental foi o fatorial 2 $\mathrm{x} 2$, e os resultados foram analisados por meio de comparações de médias (teste de Tukey).

\section{RESULTADOS E DISCUSSÃO}

Houve queda significativa na Unidade Haugh (Figura 1) em relação ao tempo de armazenamento. Os ovos mantidos em temperatura ambiente apresentaram maior queda do que os armazenados sob refrigeração, como era esperado. A perda de qualidade em função do tempo pode ter sido causada pela perda de $\mathrm{CO}_{2}$, provocando aumento do $\mathrm{pH}$ e da fluidificação do albúmen. Além disso, com o aumento da temperatura, o ovo transpira, intensificando a perda de $\mathrm{CO}_{\mathrm{e}}$ e água para o meio (GONZALES MATEOS \& BLAS BEORLEGUI, 1991).

Dados obtidos por BRUGALLI et al. (1998), após 48 horas de oviposição, indicaram que os ovos de galinha armazenados em geladeira apresentam melhor qualidade interna do que os ovos armazenados em temperatura ambiente. Segundo SILVA(2006), ocorre perda de qualidade de ovos, expressa em unidade Haugh e altura do albúmen, ao longo do armazenamento, independentemente do ambiente armazenado. No entanto, a velocidade de queda na qualidade do ovo é mais acentuada para os ovos armazenados em ambiente sem refrigeração. ALLEONI \& ANTUNES (2001) também observaram diminuição mais acentuada na altura do albúmen em ovos armazenados a $25^{\circ} \mathrm{C}$, quando comparados àqueles armazenados a $8^{\circ} \mathrm{C}$.

No presente estudo, observou-se perda gradativa de Unidades Haugh com o tempo de armazenamento e esta perda foi mais acentuada em temperatura ambiente. Entretanto, observou-se que a unidade Haugh deve ter valores de referência para ovos de avestruzes diferentes dos usados para ovos de galinha, pois o valor máximo de Unidade Haugh não pode ultrapassar 100. Neste estudo, o maior valor encontrado para unidade Haugh foi 110. Portanto, ficou acima da maior referência para ovo de galinha. Outra evidência constatada foi que, no $28^{\circ}$ dia de armazenamento, em ambiente refrigerado, os ovos apresentaram unidade Haugh de 97,70, sendo classificados como de excelente qualidade, embora mostrassem algumas evidências de deterioração.

Houve interação do tempo e da temperatura na perda de peso dos ovos de avestruzes durante o armazenamento. Constatou-se uma crescente perda de peso ao longo do armazenamento, tanto sob temperatura ambiente, quanto sob refrigeração, porém a perda de peso foi mais evidente na temperatura ambiente. Nos três primeiros dias de armazenamento, a perda foi da ordem de $0,3 \%$, seguindo-se 1,$47 ; 2,57 ; 3,8$ e 4,94\% de perda de peso para sete, 14, 21 e 28 dias de armazenamento, a $25^{\circ} \mathrm{C}$, respectivamente. Já a perda de peso em temperatura refrigerada, ocorreu de maneira menos significativa 1,2; 1,6; 2,5 e 3,6\% para os seguintes dias sob refrigeração sete, 14, 21 e 28. SINGH \& PANDA (1990) avaliaram a perda de peso em ovos armazenados a $5 \pm 1^{\circ} \mathrm{C}$ e a $32 \pm 2^{\circ} \mathrm{C}$ e confirmaram que a perda de peso 


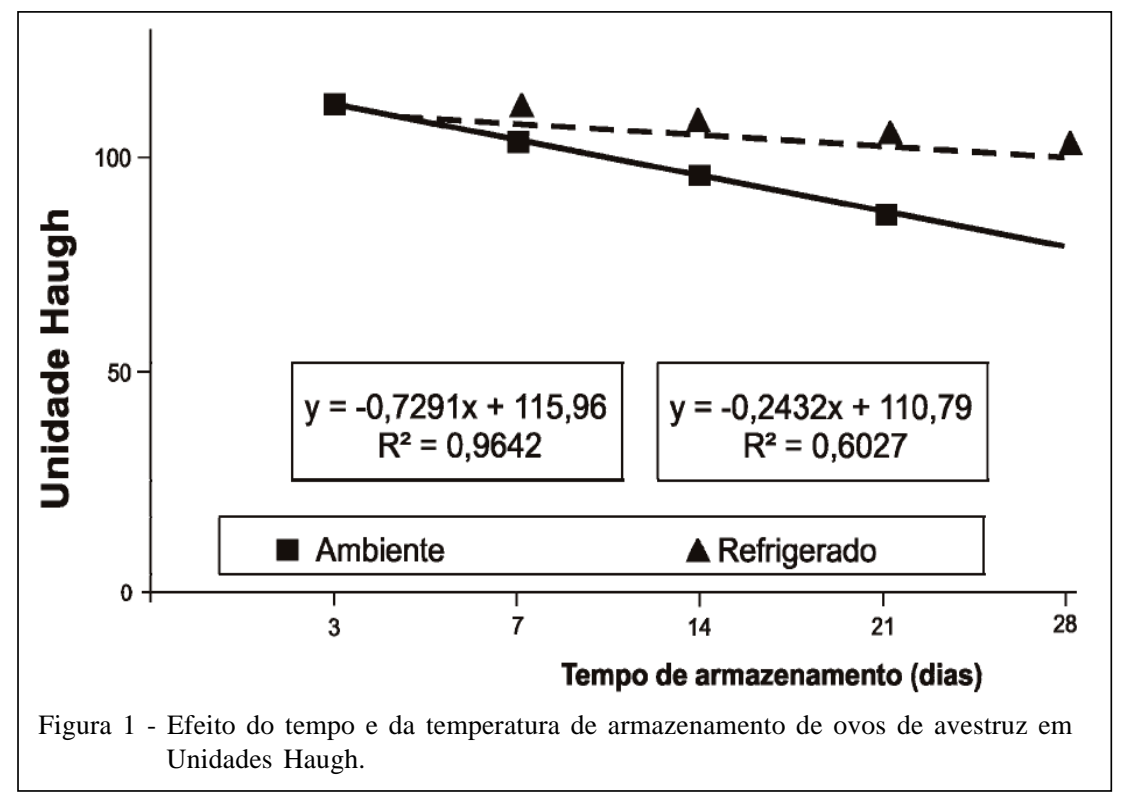

foi mais acentuada em ovos armazenados em temperatura ambiente. A perda de peso em ovos armazenados a $32 \pm 2^{\circ} \mathrm{C}$ foi de $3,57 \mathrm{~g}$ após sete dias, alcançando 9,25g em 21 dias de armazenamento. Para ovos armazenados a $5 \pm 1{ }^{\circ} \mathrm{C}$, após 14 dias, a perda de peso foi de 2,16g e, após oito semanas, foi de 10,03g. CEPERO et al. (1995) encontraram perda de peso de 1,5g, ou seja, 2,2\% para ovos armazenados durante 28 dias sob temperatura de $4^{\circ} \mathrm{C}$. Para os ovos armazenados a $18^{\circ} \mathrm{C}$, ocorreu perda de $3,5 \mathrm{~g}(5,1 \%)$, principalmente após 14 dias de armazenamento. Os ovos armazenados a $32^{\circ} \mathrm{C}$ perderam cerca de $8,5 \mathrm{~g}(12,5 \%)$, e cerca de $40 \%$ dessa perda de peso ocorreu na primeira semana de armazenamento. Durante o armazenamento, ocorre a perda de peso em ovos, devido à transferência de umidade do albúmen para o ambiente externo, por meio da casca (AHN et al., 1997; SCOTT \& SILVERSIDES, 2000; SILVERSIDES \& BUDGELL, 2004; FARIA et al., 2007).

Ao longo do tempo de armazenamento em ambas as temperaturas, foi evidenciado o aumento de $\mathrm{pH}$, o qual apresentou elevação mais evidente na temperatura ambiente (Figura 2). O pH do albúmen aumenta com a perda de CO do ovo (BURLEY \& VADEHRA, 1989) e também quando aumenta a temperatura de armazenamento (GOODRUM et al., 1989). SILVERSIDES \& SCOTT (2001) concluíram que o pH do albúmen aumenta com o tempo de estocagem, mas não aumenta com a idade do animal.

Conforme se verifica na figura 3 , houve decréscimo da viscosidade da clara em relação ao tempo

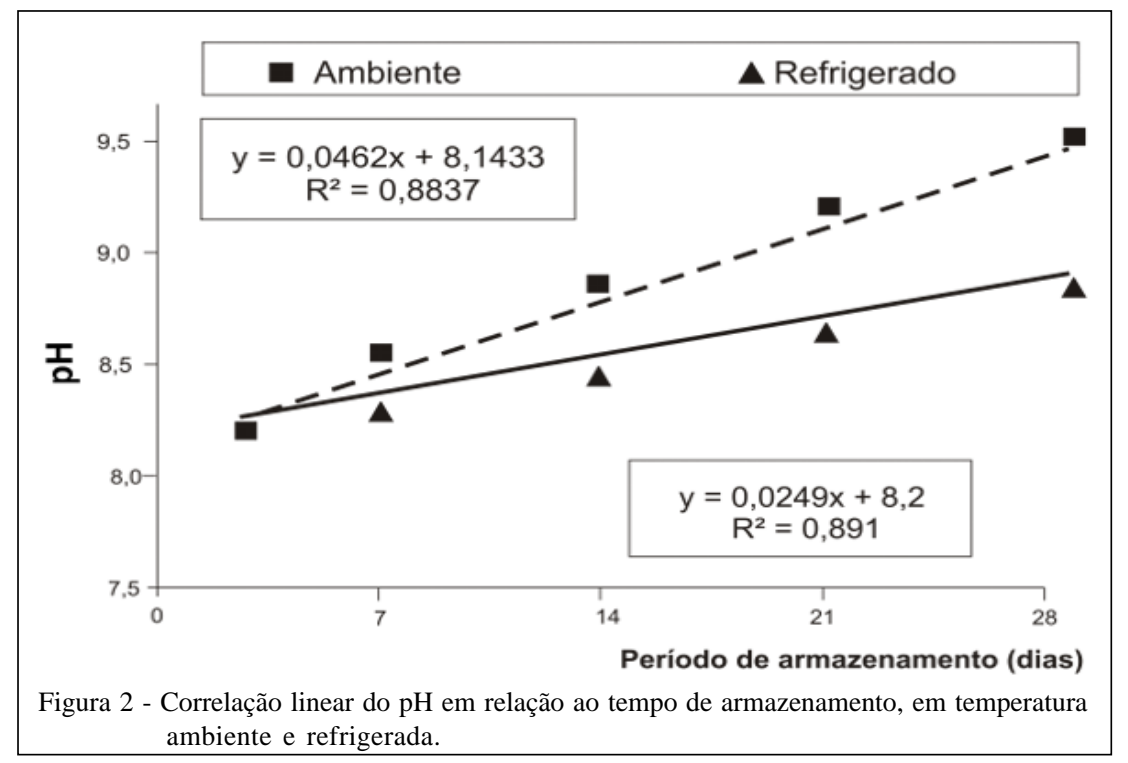

Ciência Rural, v.39, n.6, set, 2009. 


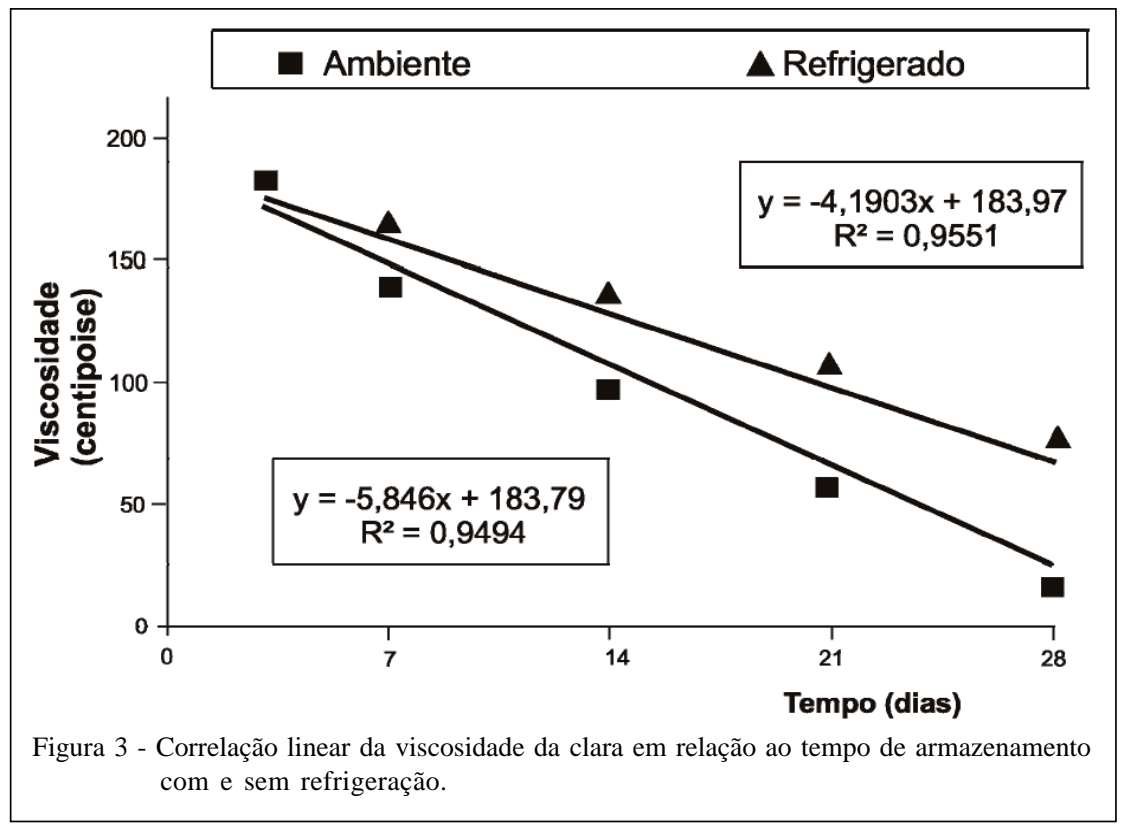

de armazenamento, e o fator temperatura também foi importante, pois foi observada diminuição mais acentuada da viscosidade da clara nos ovos armazenados em temperatura ambiente. A viscosidade inicial foi de 183 centipoise para três dias de armazenamento, reduzindo para 74,1 em 28 dias de armazenamento, em temperatura refrigerada, e para 23,9 centipoise após 28 dias, em temperatura ambiente. A queda foi de $60 \%$ da viscosidade no período de 28 dias de armazenamento, em temperatura refrigerada, enquanto que, na temperatura ambiente, a queda foi de 87\% no mesmo período.

SILVERSIDES \& SCOTT (2001), estudando o tempo de estocagem dos ovos, observaram perda progressiva na qualidade dos ovos com o passar dos dias (um a 10 dias de avaliação). Os autores observaram alterações no peso dos ovos e nas porcentagens de casca, albúmen e gema. A altura e o pH do albúmen também sofreram queda na qualidade com o passar do tempo. De acordo com FERNANDES et al. (1993), a deterioração da qualidade dos ovos é uma função direta do tempo de armazenamento, e a temperatura é fator primordial na armazenagem dos ovos para consumo, de maneira a reduzir a perda de qualidade interna e preservar o seu valor nutricional. À temperatura de geladeira $\left(5^{\circ} \mathrm{C}\right)$, a perda da qualidade interna do ovo ocorre mais lentamente em níveis inferiores àqueles ocorridos quando os ovos são mantidos em temperatura ambiente $\left(25^{\circ} \mathrm{C}\right)$.

\section{CONCLUSÃO}

A estocagem sob temperatura ambiente reduz a qualidade interna de ovos de avestruz. A refrigeração é eficaz e aumenta a vida de prateleira de ovos de avestruz. $\mathrm{O}$ ovo de avestruz, na temperatura de refrigeração, manteve boa qualidade interna, podendo ser armazenado por mais tempo. Constatouse que até 21 dias de armazenamento, em temperatura ambiente (23 a $28^{\circ} \mathrm{C}$ ), os ovos mantiveram a qualidade interna requerida para o consumo.

\section{REFERÊNCIAS}

AHN, D.U. et al. Effect of egg size and strain and age of hens on the solids content of chicken egg. Poultry Science, v.76, p.914-919, 1997.

ALLEONI, A.C.C.; ANTUNES, A.J. Unidade Haugh como medida da qualidade de ovos de galinha sob refrigeração. Sciencia Agrícola, v.58, n.4, p.681-685, 2001. Disponível em: http://www.scielo.br. Acesso em: 05 de fev. 2008. Doi: 10.1590/S0103-90162001000400005.

BERARDINELLI, A et al. Effects of transport vibrations on quality indices of shell eggs. Biosystems Engineering, v.86, n.4, p.495502, 2003. Disponível em: http://www.sciencedirect.com. Acesso em 05 de fev. 2008. Doi:10.1016/j.biosystemseng.2003.08.017.

BRANT, A.W.et al Recommended standard for scoring and measuring opened egg quality. Food Technology, v.5, p.356, 1951.

BRASIL. Ministério da Agricultura, Pecuária e Abastecimento. Regulamento de Inspeção Industrial e Sanitária de Produtos de Origem Animal. Portaria $n^{\circ} 01$, de 21 de fevereiro de 1990.

Ciência Rural, v.39, n.6, set, 2009. 
BRUGALLI, I. et al. Efeito dos níveis de óleo e proteína da dieta sobre a qualidade interna de ovos, em diferentes condições e tempo de armazenamento. Revista Brasileira de Agrociência, v.4, n.3, p.187-190, 1998.

BURLEY, R.W.; VADEHRA, D.V. The avian chemistry and biology. New York: John Wiley \& Sons, 1989. 472p.

CARRER, C.C. et al. A Criação do avestruz: guia completo de A a Z. São Paulo: OSTRICH DO BRASIL ,2004. 256p.

CEPERO, R. et al. Effects of transport and storage conditions on the commercial quality of eggs. In: BRIZ, R.C. Egg and egg products quality. Zaragoza: Acribia, 1995. 429 p.

EISEN, E.J. et al. The Haugh unit as a measure of egg albumen quality. Poultry Science, v.41, p.1461-1468, 1962.

FARIA, D.E. et al. Interação nutrição e qualidade de ovos para processamento industrial. Capturado em 27 out. 2007. On line. Disponível em: http://www.lisina.com.br/upload/ bibliografia/ovos_CBNA.2002.pdf

FERNANDES, E.A. et al. Efeito da temperatura do período de armazenamento sobre a qualidade interna dos ovos para consumo. Informe Agropecuário, v.9, p.107, 1993.

GONZALES MATEOS, G.; BLAS BEORLEGUI, C. Nutricion y alimentacion de gallinas ponedoras. Madrid: MundiPrensa, 1991. 263p.

GOODRUM, J.W et al. Effect off storage condictions on albumen $\mathrm{pH}$ and subsequent hard-cooked eggs peelabity and albumen sheer strength. Poultry Science, v.8, p.1226-1231, 1989.

LINDEN, G.; LORIENT, D. Bioquímica agroindustrial. Revalorización alimentaria de la producción agrícola. Zaragoza: Acribia, 1996. 428p

PEEBLES, E.D. et al. Effects of breeder age and dietary fat source and level on broiler hatching egg characteristics. Poultry Science, v.79 p. 698-704, 2000.
SCOTT, T.A.; SILVERSIDES, F.G. The effect of storage and strain of hen on egg quality. Poultry Science, v.79, p.17251729, 2000.

SILVA, M.F.R. Desempenho, qualidade dos ovos e balance de nitrogênio de poedeiras comerciais alimentadas com diferentes níveis de proteína bruta, metionina e lisina. 2006. 107f. Tese (Doutorado em Zootecnia) - Curso de Pós graduação da Faculdade de Zootecnia e Engenharia de Alimentos da Universidade de São Paulo.

SILVERSIDES, F.G.; SCOTT,T.A. Effect of storage and layer age on quality of eggs from two lines of hens. Poultry Science, v.80, p. 1240-1245, 2001.

SILVERSIDES, F.G.; BUDGELL, K. The relationships among measures of egg albumen height, $\mathrm{pH}$, and whipping volume. Poultry Science, v.83, p.1619-1623, 2004.

SINGH, R.P.; PANDA, B. Comparative study on some quality attributes of quail and chicken eggs during storage. Indian Journal of Animal Sciences, v.60, n.1, p.114-117, 1990.

SOUZA, H.B.A. et al. Efeito da qualidade da casca e higienização com diferentes concentrações de hipoclorito de sódio na manutenção da qualidade interna de ovos de consumo. Alimentos e Nutrição, v.5, p.27-36, 1994

SOUZA, H.B.A. et al. Influência de diferentes tipos de embalagens e tratamento com óleo mineral sobre a qualidade de ovos de consumo. In: CONGRESSO BRASILEIRO DE CIÊNCIA E TECNOLOGIA DE ALIMENTOS, 1998, Rio de Janeiro. Anais... Rio de Janeiro: SBCTA, 1998. Trabalho n. 281.

WILLIAMS, K.C. Some factors affecting albumen quality whit particular reference to Haugh unit score. World's Poultry Science Journal, v.48, p.5-16, 1992. Disponível em: http:/ /journals.cambridge.org. Acesso 30 de agosto de 2008. Doi:10.1079/WPS19920002. 